\title{
ITERATION THEORY AND INEQUALITIES FOR KLEINIAN GROUPS
}

\author{
F. W. GEHRING AND G. J. MARTIN
}

1. Introduction. An important problem in the theory of discrete groups is to decide when two Möbius transformations $f, g$ acting on the Riemann sphere $\overline{\mathbf{C}}$ generate a Kleinian group, that is, a discrete group whose limit set contains more than two points. (See [Be and M1] for further information on such groups.) Solutions to the above problem have quite general applications, for example, to deformation theory, discreteness of limits [J1] and universal constraints for Kleinian groups [Be], and lower bounds for the volume of hyperbolic manifolds [Me, W].

We report here on a connection between this problem and iteration theory [GM1]. In particular, by analyzing the stable region $D$ for a certain quadratic polynomial $R$, we find inequalities which must be satisfied by the generators of a Kleinian group. These include a stronger form of Jørgensen's inequality and inequalities new even for the Fuchsian case.

Our method is similar to that of Zassenhaus [Z], Shimizu [S], Leutbecher [Le], Jørgensen [J1], Brooks and Matelski [BM] and others. We examine a sequence of subgroups defined by iterating the commutator of the generators; after normalization the traces of the commutators of successive subgroups are related by a quadratic polynomial $R$. If the trace of the commutator of the original group lies in the region $D$, then a detailed analysis yields a convergent sequence of elements contradicting discreteness. The main difference in our approach is one of emphasis. Earlier results were obtained by looking for conditions which guarantee the existence of such a sequence. We study the region $D$ and let its geometry dictate what the hypotheses should be.

2. Main result. For $\beta \in \mathrm{C}$ we set $R_{\beta}(z)=z^{2}-\beta z$ and let $R_{\beta}^{n}$ denote the $n$th iterate of $R_{\beta}$. The filled in Julia set for $R_{\beta}$ is the bounded perfect set

$$
D(\beta)=\left\{z \in \mathbf{C}:\left\{R_{\beta}^{n}(z)\right\} \text { is a bounded sequence }\right\},
$$

and the set of eventually periodic points which do not orbit onto 0 is

$$
P^{*}(\beta)=\left\{z \in \mathrm{C}:\left\{R_{\beta}^{n}(z)\right\} \text { is a finite set not containing } 0\right\} .
$$

(See the expository articles of [Bl and Ly] for further background on this subject.)

Received by the editors July 29, 1988 and, in revised form, January 3, 1989.

1980 Mathematics Subject Classification (1985 Revision). Primary 30F40, 30D50.

The research of both authors was supported in part by the National Science Foundation, Grants DMS-87-02356 and DMS-86-02550. 
A Möbius transformation $f$ is elliptic, parabolic, hyperbolic or loxodromic if $f$ is respectively conjugate to a rotation, a translation, a homothety or a homothety plus a possible rotation. Given Möbius transformations $f$ and $g$ we introduce the parameters

$$
\gamma=\gamma(f, g)=\operatorname{tr}([f, g])-2, \quad \beta=\beta(f)=\operatorname{tr}^{2}(f)-4,
$$

where $[f, g]$ denotes the commutator $f g f^{-1} g^{-1}$ and $\operatorname{tr}(f)$ the trace of the matrix representing $f$ in $\operatorname{SL}(2, \mathbf{C})$. Then $f$ is elliptic if $\beta \in[-4,0)$, parabolic or the identity if $\beta=0$, hyperbolic if $\beta \in(0, \infty)$ and loxodromic if $\beta \notin[-4,0]$. (See [Be and M1].) If we set

$$
\Theta_{f}(g)=g f g^{-1}, \quad \Theta_{f}^{n+1}(g)=\Theta_{f}\left(\Theta_{f}^{n}(g)\right), \quad \gamma_{n}=\gamma\left(f, \Theta_{f}^{n}(g)\right),
$$

then $\gamma_{n+1}=R_{\beta}\left(\gamma_{n}\right)$ and $\Theta_{f}^{n}(g) f^{-1}=[\cdots[[g, f], f] \cdots f]$.

The following is our main result for the case where $f$ is loxodromic; similar versions hold when $f$ is elliptic or parabolic.

THEOREM 1. Suppose that $\langle f, g\rangle$ is Kleinian. If $f$ is loxodromic, then $\gamma \notin D(\beta) \backslash P^{*}(\beta)$. Moreover if $\gamma \in P^{*}(\beta)$, then there is a nontrivial relation in $\langle f, g\rangle$ of one of the following types

$$
\Theta_{f}^{n}(g)=\Theta_{f}^{m}(g), \quad n \geq m+2 \text { or } f^{k} \Theta_{f}^{n}(g) f^{-k}=\Theta_{f}^{m}(g), \quad k \neq 0 .
$$

The polynomial $R_{\beta}$ is conjugate by $m(z)=z-\beta / 2$ to $p(z)=z^{2}+c$ where

$$
c=c(\beta)=\frac{1}{4}\left(1-(\beta+1)^{2}\right) .
$$

The Mandelbrot set $\mathbf{M}$ is the set of $c$ for which the filled in Julia set $D(\beta)$ has a connected boundary. Generically then, if $c$ lies in $\mathbf{M}, \operatorname{int}(D(\beta))$ will be nonempty and we obtain an inequality for $(\gamma, \beta)$ which must be satisfied in order that $\langle f, g\rangle$ be Kleinian.

For example, the two fixed points for $R_{\beta}$ are 0 and $\beta+1$. If $|\beta|<1$, then 0 is attracting, all eventually periodic fixed points in $\operatorname{int}(D(\beta))$ must eventually orbit onto 0 and hence $P^{*}(\beta) \cap \operatorname{int}(D(\beta))=\varnothing$. Next

$$
R_{\beta}\left(B^{2}(0,1-|\beta|)\right) \subset B^{2}(0,1-|\beta|) \text { whence } B^{2}(0,1-|\beta|) \subset D(\beta) .
$$

The symmetry of $R_{\beta}$ about its critical point $\beta / 2$ implies that $z \in D(\beta)$ if and only if $\beta-z \in D(\beta)$. These facts yield the following result.

Corollary 1. Suppose that $\langle f, g\rangle$ is Kleinian. Then

$$
|\gamma|+|\beta| \geq 1 \text { and }|\gamma-\beta|+|\beta| \geq 1 \text {. }
$$

Both inequalities are due to Jørgensen, who obtained the first by iteration and the second from the first using the Lie product $[\mathbf{J 1}, \mathbf{J} 2]$. Indeed, the symmetry property of $R_{\beta}$ about $\beta / 2$ can, in turn, be used to define this product [GM1].

When $f$ is not parabolic, the set $B^{2}(0,1-|\beta|) \cup B^{2}(\beta, 1-|\beta|)$, excluded for $\gamma$ in Corollary 1 , generally constitutes only a small portion of the set $D(\beta)$. See, for example, Figure 1 . 


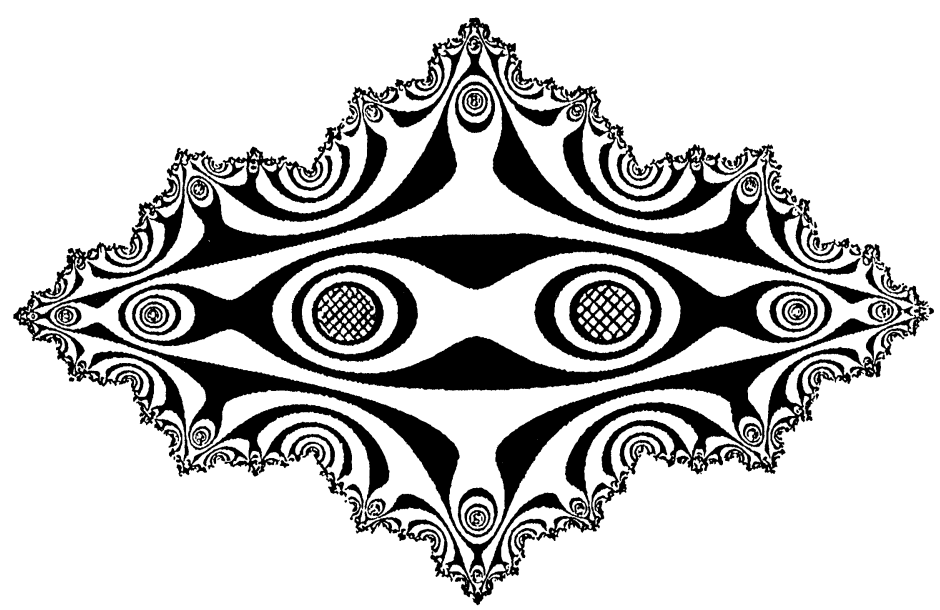

(a)

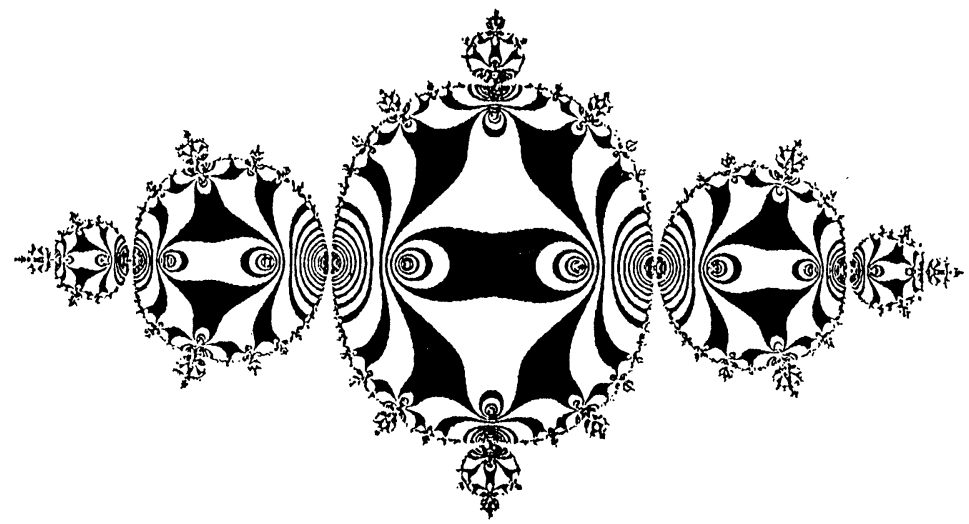

(b)

\section{Figure 1}

The filled in Julia sets $D(\beta)$ for (a) $\beta=0.8$ and (b) $\beta=1.048$. The banding indicates the iteration scheme. In (a) $P^{*}(\beta)$ consists only of a countable dense subset of the boundary. The cross hatched disks are those given by Jørgensen's inequalities. Notice the symmetry about the critical point. In (b) $P^{*}(\beta)$ consists of a countable dense subset of the boundary together with those points at which the banding accumulates. The set (b) comes from the $(2,3,7)$ Triangle group whose parameters are those of the period two attracting cycle. Notice in (b) Jørgensen's inequality yields no information as $|\beta|>1$. 
The inequalities in Corollary 1 are sharp [JK]; they hold with equality only when $\bar{B}^{2}(0,1-|\beta|)$ meets the Julia set $\partial D(\beta)$ in an eventually periodic point. This fact yields a conceptually simple proof of the stability and structure of groups for which either of these inequalities holds with equality [GM2].

Theorem 1 does not exclude the possibility that $\gamma \in P^{*}(\beta) \subset D(\beta)$; hence there are exceptions to a general analysis about the attractive periodic cycles. We say that a Kleinian group $\langle f, g\rangle$ is extremal if $\gamma \in P^{*}(\beta)$ and exceptional if $\gamma \in P^{*}(\beta) \cap \operatorname{int}(D(\beta))$. Both classes of groups exist. Since the eventually periodic points are isolated in $\operatorname{int}(D(\beta))$, we see for purely combinatorial reasons that these groups are quite isolated in the space of all discrete groups [GM3]. Mañé, Sad and Sullivan show that generically the structure of the filled in Julia set is stable under perturbation [MSS]; this also implies certain rigidity properties of exceptional groups.

The relations in Theorem 1 yield algebraic and geometric properties of extremal groups and show this apparently combinatorial property is a homomorphic invariant of the group. For example, the only exceptional Fuchsian groups are the $(2,3,7)$ and $(2,4,5)$ triangle groups. Among the extremal groups are the $(3,3,4)$ and $(2,3, p)$ triangle groups, some knot complements and some compact hyperbolic three manifolds that fiber over the circle [J3].

When $|\beta+2|<1, \beta+1$ is an attracting fixed point for $R_{\beta}$. An analysis of the exceptional groups yields the following result.

Corollary 2. Suppose that $\langle f, g\rangle$ is Kleinian. Then

$$
|\gamma-\beta-1|+|\beta+2| \geq 1 \text { and }|\gamma+1|+|\beta+2| \geq 1
$$

unless $\gamma=\beta+1$ in the first inequality or $\gamma=-1$ in the second.

This result was also discovered recently by Tan [T]. The following inequalities result from a study of the critical point $\beta / 2$.

Corollary 3. Suppose that $\langle f, g\rangle$ is Kleinian. If $|\beta(\beta+2)| \leq 1$ and if $\gamma \neq \beta+1$ and $\gamma \neq-1$, then

$$
|2 \gamma-\beta| \geq 1+(1-|\beta(\beta+2)|)^{1 / 2} .
$$

If $|(\gamma+2)(\gamma+4)| \leq 1$ and if $\gamma \neq \beta+1$ and $\beta \neq-3$, then

$$
|2 \beta-\gamma+4| \geq 1+(1-|(\gamma+2)(\gamma+4)|)^{1 / 2} .
$$

3. Totally real polygon. Theorem 1 and its analogues exclude a region of $\mathbf{C}^{2}$ for the parameters of nonexceptional Kleinian groups. When $\gamma$ and $\beta$ are real, precise calculations can be made; this does not mean restricting ourselves to the Fuchsian case. In particular, if $\beta \in[-4,2]$, then $c=c(\beta)$ lies in the Mandelbrot set $\mathbf{M}$ and the intersection of $D(\beta)$ with the real axis is the interval $I(\beta)=[\min (-1, \beta), \max (0, \beta+1)]$. Hence the polygon

$$
\Omega=\{(\gamma, \beta): \gamma \in I(\beta), \beta \in[-4,2]\}
$$

contains no parameters for $\langle f, g\rangle$ unless it is extremal. See Figure 2. 


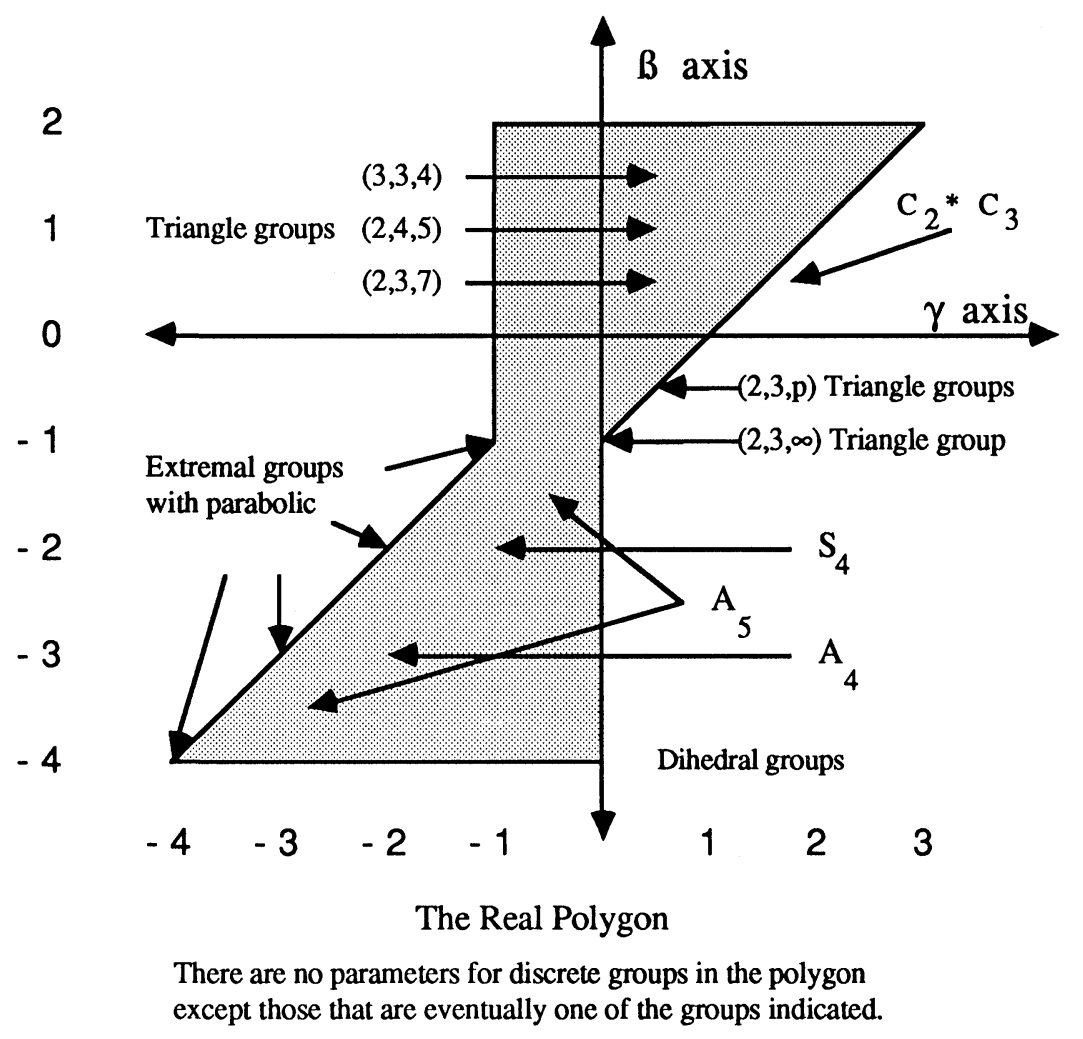

FIGURE 2

Theorem 2. Suppose that $\langle f, g\rangle$ is Kleinian and that $(\gamma, \beta)$ lies in $\operatorname{int}(\Omega)$. If $f$ is hyperbolic, then

$$
\beta=2(\cos (2 \pi / 7)+\cos (\pi / 7)-1) \quad \text { or } \beta=\sqrt{5}-1 \quad \text { or } \beta=2 \sqrt{2}-1 \text {, }
$$

in which case $\left\langle f, g \mathrm{fg}^{-1}\right\rangle$ is respectively the $(2,3,7),(2,4,5),(3,3,4)$ triangle group. If $f$ is elliptic, then

$$
\beta=-2 \text { or } \beta=-3 \text { or } \beta=-(5 \pm \sqrt{5}) / 2
$$

in which case $\left\langle f, g f g^{-1}\right\rangle$ is respectively $S_{4}, A_{4}$ or $A_{5}, A_{5}$.

RemarK 1. With these exceptions, no Kleinian group $\langle f, g\rangle$ has parameters in int $(\Omega)$. On the other hand, there are many groups with parameters in $\partial \Omega$. For example, each integer lattice point in $\partial \Omega$ corresponds to a discrete group which is also nonelementary whenever $\gamma \neq 0$. Moreover, the $(2,3, p)$ triangle groups correspond to points in $\partial \Omega$ which accumulate at the point $(0,-1)$ corresponding to the $(2,3, \infty)$ group. That the groups with $(\gamma, \beta)=(-n,-n), n=1,2,3,4$, are discrete follows from recent results of Maskit [M2].

REMARK 2. Theorems 1 and 2 yield necessary conditions for the parameters $(\gamma, \beta)$ of a Kleinian group $\langle f, g\rangle$. Other conditions result from 
looking at parameters corresponding to subgroups, new generators or the Lie product [T]. For example, let $\sigma$ be an element of the group generated by the reflections

$$
\phi(\gamma, \beta)=(\gamma, \gamma-\beta-4), \quad \psi(\gamma, \beta)=(\beta-\gamma, \beta),
$$

a group isomorphic to $S_{3}$. Then the parameters $(\gamma, \beta)$ are admissible for a discrete group $\langle f, g\rangle$ if and only if the parameters $\sigma(\gamma, \beta)$ are. This fact yields four additional pairs of inequalities, equivalent to those in Corollaries 1 and 2, and a larger excluded region in Theorem 2.

5. Geometric estimates. The inequalities above contain geometric information about the Kleinian group $\langle f, g\rangle$. For example, those in Corollary 1 imply that $f$ and $g$ cannot both be close to the identity. We conclude with two explicit estimates which quantify this fact. (See also [W].) The first is given in terms of the matrices $A$ and $B$, which represent $f$ and $g$ in $\operatorname{SL}(2, \mathbf{C})$, and the norm $\|C\|=\operatorname{tr}\left(C C^{*}\right)^{1 / 2}$. The second estimate involves the metric

$$
d(f, g)=\sup _{z \in \overline{\mathbf{C}}} q(f(z), g(z)) \in[0,2],
$$

where $q$ denotes chordal distance in $\overline{\mathbf{C}}$.

Theorem 3. Suppose that $\langle f, g\rangle$ is a Kleinian group with identity element id. Then

$$
\left\|A-A^{-1}\right\|\left\|B-B^{-1}\right\| \geq c_{0}, \quad \max (d(f, \mathrm{id}), d(g, \mathrm{id})) \geq d_{0},
$$

where $c_{0}$ and $d_{0}$ are absolute constants,

$$
\begin{gathered}
4(\sqrt{2}-1)=1.65 . . \leq c_{0} \leq 1.98 . .=4(2 \cos (2 \pi / 7)-1)^{1 / 2}=c_{1}, \\
2(\sqrt{2}-1)=.828 . . \leq d_{0} \leq .911 . .=2\left(\frac{\cos (2 \pi / 7)+\cos (\pi / 7)-1}{\cos (2 \pi / 7)+\cos (\pi / 7)+1}\right)^{1 / 2}=d_{1} .
\end{gathered}
$$

RemarK 3. The lower bounds for the constants $c_{0}$ and $d_{0}$ result from Jørgensen's inequality and inequalities relating $|\beta|$ to $\left\|A-A^{-1}\right\|$ and $|\gamma|$ to $\left\|A-A^{-1}\right\|\left\|B-B^{-1}\right\|$ [GM4]. The upper bounds $c_{1}$ and $d_{1}$ come from the $(2,3,7)$ triangle group. When $\langle f, g\rangle$ is Fuchsian, Theorem 3 holds with $c_{0}=c_{1}$ and $d_{0}=d_{1}$ and hence is sharp for this case.

ReMARK 4. A Möbius group $G$ is discrete if there exists a constant $d=d(G)>0$ such that $d(f, g) \geq d$ for each distinct pair $f, g$ in $G$. In this case, Theorem 3 implies there exists a Möbius transformation $h$ such that $d\left(h f h^{-1}, h g h^{-1}\right) \geq d_{0}$ for each distinct pair $f, g$ in $G$, where $d_{0}$ is as above. Thus each discrete Möbius group is conjugate to one in which distances between distinct elements are bounded below by a universal constant.

\section{REFERENCES}

[Be] A. F. Beardon, The geometry of discrete groups, Springer-Verlag, Berlin and New York, 1983.

[B1] P. Blanchard, Complex analytic dynamics on the Riemann sphere, Bull. Amer. Math. Soc. (N.S.) 11 (1984), 85-141. 
[BM] R. Brooks and J. P. Matelski, The dynamics of 2-generator subgroups of PSL $(2, \mathbf{C})$, Riemann surfaces and related topics, Ann. Math. Studies 97, Princeton Univ. Press, Princeton, N.J., 1981.

[GM1] F. W. Gehring and G. J. Martin, Discreteness in Kleinian groups and the iteration theory of quadratic mappings (to appear).

[GM2] __, Stability and extremality in Jørgensen's inequality, Complex Variables (to appear).

[GM3] _ - On the rigidity of certain triangle groups (to appear).

[GM4] __ Inequalities for Möbius transformations and discrete groups (to appear).

[J1] T. Jørgensen, On discrete groups of Möbius transformations, Amer. J. Math. 98 (1976), 739-749.

[J2] _ Comments on a discreteness condition for subgroups of $S L(2, \mathbf{C})$, Canad. J. Math. 31 (1979), 87-92.

[J3] _ Compact 3-manifolds of constant negative curvature fibering over the circle, Ann. of Math. (2) 106 (1977), 61-72.

[JK] T. Jørgensen and M. Kiikka, Some extreme discrete groups, Ann. Acad. Sci. Fenn. 1 (1975), 245-248.

[Le] A. Leutbecher, Über Spitzen diskontinuierlicher Gruppen von lineargebrochenen Transformationen, Math. Z. 100 (1967), 183-200.

[Ly] M. Yu. Lyubich, The dynamics of rational transforms: the topological picture, Russian Math. Surveys 41:4 (1986), 43-117.

[MSS] R. Mañé, P. Sad and D. Sullivan, On the dynamics of rational maps, Ann. Sci. École Norm. Sup. (4) 16 (1983), 193-217.

[M1] B. Maskit, Kleinian groups, Springer-Verlag, Berlin and New York, 1988.

[M2] __ Some special 2-generator Kleinian groups, Proc. Amer. Math. Soc. (to appear).

[Me] R. Meyerhoff, A lower bound for the volume of hyperbolic 3-manifolds, Canad. J. Math. 39 (1987), 1038-1056.

[S] H. Shimizu, On discontinuous groups operating on the product of the upper half planes, Ann. of Math. 77 (1963), 33-71.

[T] D. Tan, On two generator discrete groups of Möbius transformations, Proc. Amer. Math. Soc. (to appear).

[W] P. L. Waterman, An inscribed ball for Kleinian groups, J. London Math. Soc. 16 (1984), 525-530.

[Z] H. Zassenhaus, Beweis eines Satzes über diskrete Gruppen, Abh. Math. Sem. Univ. Hamburg 12 (1938), 289-312. 48109

Department of Mathematics, University of Michigan, Ann Arbor, Michigan

Department of Mathematics, Yale University, New Haven, Connecticut 06520 
